\title{
Mendelian susceptibility to mycobacterial diseases due to partial STAT1 deficiency
}

INSERM

\section{Source}

INSERM. (1999). Orphanet: an online rare disease and orphan drug data base. Mendelian susceptibility to mycobacterial diseases due to partial STAT 1 deficiency. ORPHA:319595

Mendelian susceptibility to mycobacterial diseases (MSMD) due to partial STAT1 (signal transducer and activator of transcription 1) deficiency is a genetic variant of MSMD (see this term) characterized by a partial defect in the interferon (IFN)-gamma pathway, leading to mild mycobacterial infections. 